\title{
New Media Semiotics and the Rise of Universal Symbolic Language: Digital Natives' Perspective Amidst Covid-19
}

\author{
Emmanuel Ezimako Nzeaka \\ Pan-Atlantic University Lagos Nigeria
}

Speech is cultural identity. This peculiarity is challenged by symbolic media languages such as emoticons, emojis, memes, and diacritical computer symbols. The distinction between language and universal phonological dialect among digital natives is related by their specific use of computer symbols in media communication. Consequently, a new modality of linguistic processes is emerging, which demolishes the barriers of distinct languages. The research used the Semiotic Theory and tested 267 adolescents aged 15 to 20 years. Data were collected via a quasi-structured questionnaire, purpose and snowball sampling, 95\% confidence level. The results indicated semiotics as a significant communication function for digital natives. It draws themes and concluded that, despite its preponderance in communication, semiotics does not challenge to formal writing. Important majority $n=133, S D=12.03$ indicated that semiotics may become global language. This study shows that humanity is increasingly rallying to the use of a common language by symbols, as before the emergence of distinctive expression. This area is of importance to researchers and policy makers in a single human language evolution.

Keywords: semiotics, language evolution, emojis, emoticons, digital natives

\section{INTRODUCTION}

The intensification in recent times of the prominence of symbolic language (Semiotics) in words, letters and speeches of this generation of new media users has drawn the attention of researchers for many years now. Semiotics could be suggested through the pictorial, acoustic, perceptible, olfactory and gesticulatory means to another person beyond the conventional mode of communication (Norquist 2020). According to Umbeco Eco (1979) 'semiotics is concerned with everything that can be taken as a sign. A sign is everything which can be taken as significantly substituting for something else' (p. 9); something else may mean something that has not been in existence before the symbol. Similar work has also been pursued by others (Proir,2014) in which he gave a broad definition as

Semiotics is a broad, diverse field that involves the study of multiple kinds of signs conveyed via varied channels and media, of socially-organised and evolutionarilygenerated sign systems, and of the conditions of signification or semiosis (i.e., the processes of making meaning from signs) (p.1.)

Both definitions are closely affiliated and have a common perception of the description of the term. The application of this reliable way of communication is in no doubt, putting pressure on the traditional 
means of human interaction, especially as it relates to the new media messages between the youths and university students. Some schools of thought have commented on this rapid development that has been going on for some time as a negative development that could endanger the normal human mode of communication and hinder the advancement of human language evolution as has been evidenced in various written texts and research.

However, other researchers envision the intrusion into human speech as a positive development. They base their argument on the premise that the development would globally break down language and cultural hindrances occasioned by diverse tongues, thereby unifying human linguistic development and bring the world closer to the use of one universal language. That is to say, a person in Nigeria could communicate with a Japanese and have a full understanding of a transaction without a translator or machine assistance. The current situation has developed so pervasively that it has been grafted into the culture of everyday communication in speech, writing and other modes of human transmission of messages to the extent that it has become a salient part of healthy interaction of the millennials in both academic communiqué (sometimes) and everyday social-communicative engagements.

In research circles, the emergence of symbolism in regular communication is giving cause for concern because if the abrupt intrusion and application, especially in the written communication of the younger generation. This way of complementing which has now become a significant challenge was there before the emergence of the new internet writing itself originated in the diacritic formats of the ancient Igbo Nsibidi, the cuneiform, hieroglyphics and the Mayan graphic communication patterns. The resurgence of this types modern semiotics has been made prominent with the arrival of the internet and the new media which has given more extensive freedom to people to write in the way they deem fit for a universal application. In Nigeria, in particular, the resurgence of this medium of communication has created many challenges for the conservative rules of engagement in writing because Nigeria is considered a second language English speaking country.

Consequently, the resurgence of symbolic writing is viewed by scholars and concerned citizens as an extra burden considering that we are still learning the ropes of a second language English. The vast majority of scholars see this as a significant interjection to the writing and communicating in the English Language. In the schools, especially the higher institutions in Nigeria, student are so versed in it that it has started seeping into their official communication and writing network and some of them are quick to defend the usage as necessary. This issue, at times, creates a conflict of interest on which communication mode should be accepted as correct in the school environment. Though we know that they are not permissible in official /formal communication, some diacritics like LOL, HBD, are gradually being incorporated into formal communication in institutions, especially between students and teachers. The impact is overwhelming in a typical school environment in these days of Covid-19 when lessons have moved online, and teachers use media applications in their teaching sessions like WhatsApp, Zoom, Facebook, Instagram and many more to engage the students in active teaching and learning. The propensity is very high for the student to deploy symbolic writing and for the teacher to accept them as usual, especially in informal communication. These explanations are relevant because the use of symbol has become a reality in everyday conversation beyond regular writing. After all, the pattern is gradually but steadily gaining traction in both official and unofficial communication in writing, advertisement, speech, dressing and other modes of human interaction.

At the pace of this nascent development, the question will soon arise whether to incorporate these diacritics and symbols as part of our official communication. Are we to abandon varied languages and cultures that make us distinct, thereby creating a universal culture and communication that the human race would understand? This condition is to make the world a place unified by one language through signs and accepted symbols in intellectual and social-economic engagements. The essence is that at the level of development driven by the internet and the social media coupled with the drive of human independence and creativity, it would be too far to attain a universal language through symbols and diacritics.

In this section, we would assess the relevance of this study. The scope incorporates the interaction of the subject matter with the internet generation and university students between the age range of 15-25, who are conversant with the new media and who apply symbols in their daily writing and communication. Particular attention is paid to young adults, especially university students because they consistently use the 
internet in regular connection with various audiences where the use of short forms and symbols are prevalent. This situation would enable us to understand the motivations behind the applications and the popularity of this nature of writing. Following this system will also allow the researchers to understand why they consistently apply them in writing, how they came about it, and the convenience that they think comes with it. The impact on their social life would also be determined, thereby exposing the study of other areas of scholarship like their interest and what defines it. Besides, this will lead research to ask questions on advantages, shortcomings including how internet drives interest in this region. These areas pointed out are relevant in this study because the pace at which symbols in communication replace word calls for a concerted effort at understanding the phenomenon that is striving to unify the world in one universal language. However, this has been studied and theorised by various scholars like Pierce, Saussure, Voloshinov, Halliday, Vygotsky and a host others. The recent resurgence call for an overview by scholars. Below is an emoji essay by a student on the relevance of education.

\section{FIGURE 1 \\ AN EMOJI ESSAY FROM A STUDENT ON THE RELEVANCE OF EDUCATION}

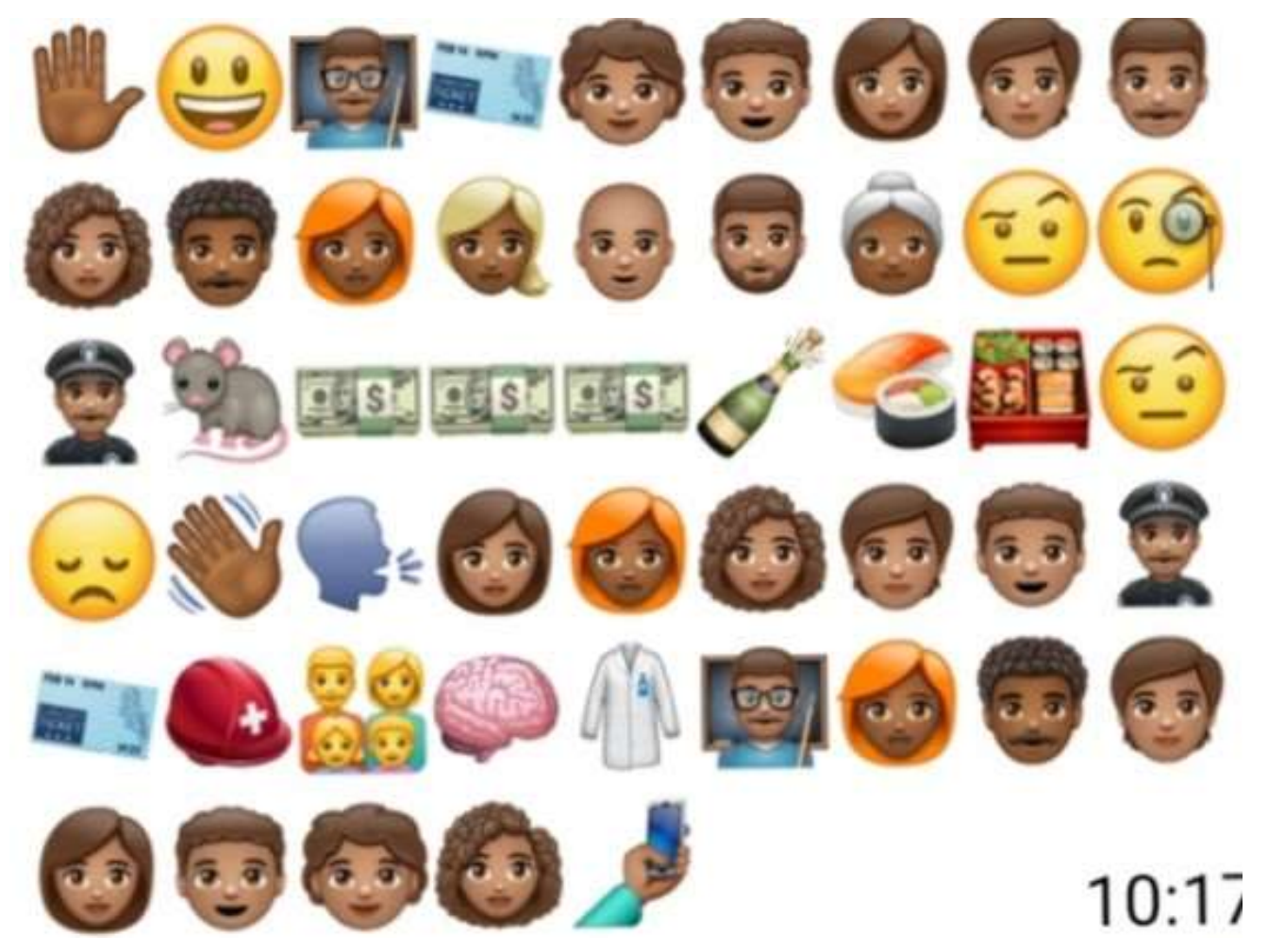

Language is an aspect of human existence and peculiar identity which has created uniqueness in human life. Regional conventions determine human communication of writing and speech that are culture-based, which has prevented the development of global language development. However, the resurgence of symbolic writing necessitated by the internet has created new challenges and making new demands of alphabetic writing, that is threatening to modify the regular written communication pattern. Scholars report the negative and positive aspect of this development. The pessimists see the advancement in diacritics as a significant development because it may break down language hindrances between cultures, thereby ushering in a universal language. Therefore, in response to this problem, our study investigates the uses and possibility of symbols advancing the course for a universal language.

The aim of the study is an exploration and examination of the use of semiotics (Emoticons, Emoji, memes) and its encroachment into formal writing mode of college students in Nigeria, especially in this 
Covid-19 era forcing a massive transition to online learning. It dealt mainly with the following specific objectives:

- The extent to which symbolic writing has permeated into formal communication patterns among the digital generations in tertiary institutions in Nigeria.

- To determine the rate, speed, penetration at which penetrated official writing and communication

- To explore the attitude of the digital generation to the use of semiotics in formal writing.

- To examine how the combination of adjunctive and subjunctive patterns in writing would advance the course for a universal language.

The following research questions have been advanced to address the objectives of the study

- To what extent has symbolic lettering intruded into the standard patterns of communication and writing?

- How has symbolic writing made communication convenient among the digital generation in Nigeria?

Therefore, in response to these problems, our study proposes to investigate specifically the use and the possibility of semiotics advancing the course for a universal language. The paper begins below by tracing the theoretical context that would guide the study. The subsequent section will present the literature review and the theoretical foundation of our investigation in the research field to understand the usages and experiences of semiotics among university students. The middle section will look at the methodology and interpretation of findings. The last part will be the recommendations and conclusion.

\section{THEORETICAL CONCEPT}

The central point of this paper is to analyse the application of symbolic language in the communication patterns of the internet generation in Nigeria and how the constant use of these diacritics has obstructed the normal communication patterns. Therefore, as a guide to the endeavour, it would critically look at the theories that would guide this study to a conclusion. The general guide will is taken from three different theorists in the field of semiotics: Ferdinand de Saussure, Charles Sanders Peirce, and Valentino Voloshinov. These are noted philosophers and forebears of symbolic language theories whose knowledge have spread beyond human communication to psychology, linguistics and philosophy. "The present shape of the field has been forged in the competing theoretical traditions of Saussure's (1983) dyadic and Peirce's (1998) triadic accounts of the sign and Voloshinov's (1973) historical-materialist accounts of the social and psychological functions of signs. "(Prior,2014,p. 1). Again, their theories are varied based on social and class conceptions and interpretations which would help the interpretation of this work from different social and cultural perspectives

\section{Theory of Sign by Ferdinand de Saussure, Charles Sanders Peirce, and Velentine Voloshinov}

Ferdinand de Saussure developed the foundation for the general linguistic theory, the theory of sign which the leading principle emphasised language as a system of signs and symbols and other diacritics that exist in humanity. In his postulations, linguistic signs are the most dominant sign pattern that exists in humanity because they play a prominent role in creating reality. He paid more attention to the underlying system of language (Langue) as against the use of language (Parole or speech). His theory placed much emphasis on the cognitive structure of the internal thought process in the determination of the physical signs of their setting. In essence, the language does not stand for reality; rather, it fabricates it because we do not give meaning to existence but also to things that do not exist. This theory, in summary, mainly looks at what constitutes signs and the laws that control them. Besides, Charles Sanders Peirce originated the pragmatism doctrine which posits that three components make up a sign: a sign, an object, and, an interpretant. For a broader understanding, we take as a singer like the written alphabet, or a spoken word, the object is whatever is signified, like the human speech, and the interpretant stands for the understanding of the combination of the sign/object relationship. The interpretation of the symbol lies with the interpretant 
to determine the interpretation and object generates. "The idea is that the interpretant provides a translation of the sign, allowing us a more sophisticated understanding of the sign's object " (Stanford Encyclopedia of Philosophy, 2016). The differences between Peirce and Saussure's orientations are on reality as well as the discipline of epistemology. For Peirce, the 'reality' lies outside the internal structure of human and is not related to each other while for Saussure, reality has a bond with our physical or human minds. Peirce questions the relationship between reality and the sign while Saussure pays attention to the association between signs which he considered as an aspect of the word creation (Masinambow \& Rahayu 2001; Yakina $\&$ Totua, 2014). There is an apparent absence of harmony among the researchers. However, it is of note that they draw motivations from the social-cultural reality of modes of communication and interpretation.

However, in a different approach, Voloshinov conceived semiotics as emanating from an amalgam of society and individual experience, which means that symbol are constructed socially, which in turn creates material reality. He insisted that, the domain of ideology coincides with the domain of signs. They equate with one another. Wherever a sign is present, ideology is present, too. Everything ideological possesses semiotic value. Every ideological sign is not only a reflection, a shadow, of reality but is also itself a material segment of that very reality. Every phenomenon functioning as an ideological sign has some kind of material embodiment, whether is sound, physical mass, colour, movements of the body, or the like (n.p.).

He pointed out that language should not be disconnected from social reality as they are common signs and symbols that have a different interpretation for different classes. Therefore, passive analysis do not interpret signs, and they are regulated by social status and parameter, which gives it a different presentation according to one's group status. This condition is a socialist approach to the understanding of symbols, which is a drastic departure from the other theories that are human-centric as the most interpretation of the symbols depended on individual interpretation. Voloshinov introduced another dimension of social class to semiotics which postulates that all words emanate from the inventory of common signs and symbols. These three theories summarised further expand our conception of semiotics from diverse schools and advanced the theory that signs and symbols are socially driven. Everything is determined as we have today, with the advancement of the internet and social media, have become embedded in our new media culture. This symbiosis has blended with the residual culture of semiotics to advance the course for the development of a universal language that will be independent of the formal ways of communication and writing.

FIGURE 2

\section{THE RELATIONSHIP BETWEEN THE THREE THEORIES. A SELF-CONCEPT}

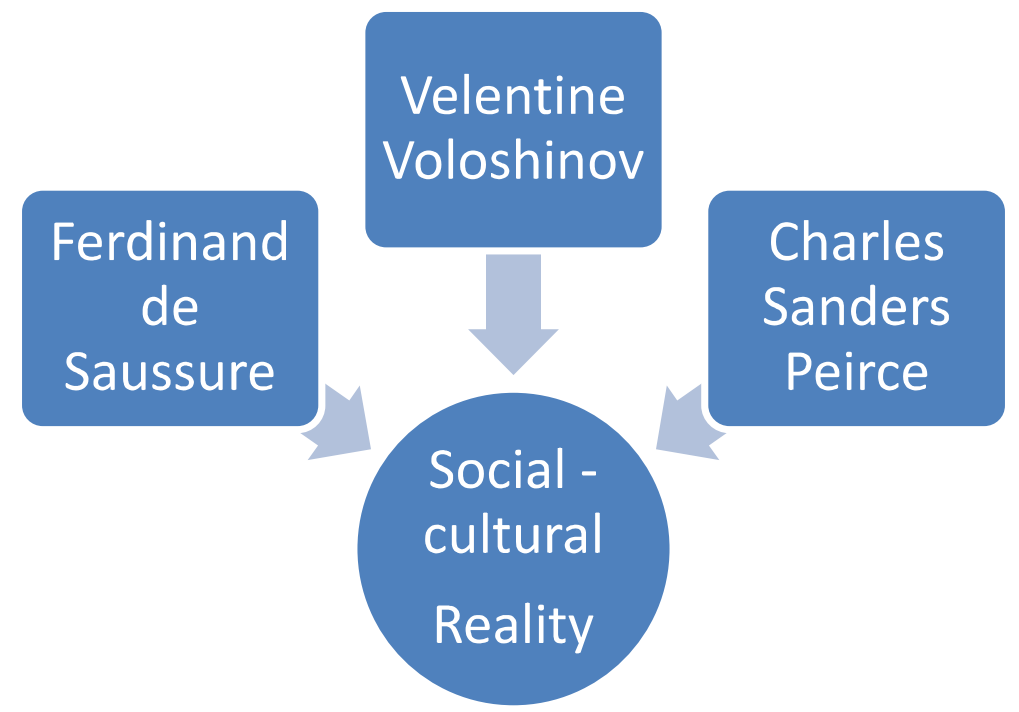

The three theories hurtle towards social reality because the comparatively draw inspiration from society to forge various approaches to understanding semiotics. 


\section{LITERATURE REVIEW}

Here we survey prior work and go on to suggest our methods. There exists an extensive literature on the topic of semiotics. Many researchers have made their views known about the influence of semiotics, emojis, emoticons, logos, brands and diacritic mark in the development of human communication. Consequently, they have drawn diverse conclusions about the development of that message form (Norquist, 2020). The use of semiotics and visual diacritic marks to represent words and feeling in writing among the internet generation in emails and other communication modes have been adjudged by some researchers as a devaluation and a rude intrusion into the conservative ways of writing and communication. Also, some other critics do not accept this as they see it as a positive development that draws inspiration from the ancient Igbo Nsibidi diacritic form to hieroglyphics to the cuneiform and other types of symbolic writings that have attempted to reduce language diversity across the generations of humanity. Semiotics seeks to return language and communication to its ancient form where images and symbols make words in a universal format (Alshenqeeti, 2016). He does on through elaborate instances to point out that symbolic writings are not new as humanity has striven since creation to have a universal language through symbolic representations as this development did not start with the advent of the internet. The internet helped in the resurgence of that mode of communication and writing that has always been there latent.

Language reacts to social situations and attitudes as humans have still needed to communicate, and in particular, connect their state of mind and sentiments, either as a defence or sharing stratagem, and through antiquity have used depictions or symbol to accomplish this and generate optical networks with one another (Fischer,2003; Alshqeeti,2016). This situation was indeed the crux of the argument by (Denesi,2016), who argued that emojis had extended human linguistic capacity to the level that it has introduced innovation to the traditional channels of communication and writing; thereby creating an expansion in the conventional ways of writing and making it more independently and creatively. This condition resonates the past connections with the ancient Egyptian, African and Sumerian symbolic writing forms. The system he pointed out has been sustained by the internet and the new media which has made it of inherent importance. The global relevance of symbolic language as a worldwide practice which transcends cultural and national barriers attract attention. Denasi points this out, what the emoji phenomenon is showing, more than anything else, is that visuality and phonetic writing are merging more and more to produce a hybrid language and, thus, that human communication in written form is evolving more and more on a single path of hybridisation across the globe, at least on the surface. We are beginning to sense, in other words, that the traditional forms of language and writing are no longer able to carry our thoughts in the ways of the past (p.88)

This recent rise in semiotics is a product of the internet age and the interrelation with human cultural consciousness and communication. In the same vein Prior() pointed that sociohistorical semiotics has begun to emerge due to the combination of psychological and cultural ideology which has developed diverse ways that human beings can communicate beyond cultural barriers in a chain of media activity. The cognisance of this dialogic connection of universal human connection and opens a critical issue of people can communicate across cultural divides across genres and other contextualisation. Prior and Hengst (2010) argued that researchers should recognise the simultaneous, layered deployment of multiple semiotics (talk, gesture, artefact use and production, interaction with environmental structure): people are never just talking, just reading, just writing. It also means that researchers should look at semiotic trajectories and chains across time and place, recognising both the need to understand semiotics as dispersed and mediated and the value of tracing out mediations ANT-like, rhizomatically, across situated functional systems (p.11)

He goes on to argue how time and distance could be complemented in this case of cross nationality of writing and communication considering the historical tradition of such exploration, that this could create a limiting factor in global sign language. However, all questions of language are concerned with semiotics. Point of diversion here is that semiotics is still evolving as a universal as a global language; this evolution has been advanced with the speed and advancement of the internet. Communication can reach out more than ever before through speech and writing to communicate more than ever before to engage in diverse ways. The freedom created by this has enabled them to develop a distinct form that could be understood across cultures without any systemic hindrance of time and space. This matter reinforces the point that 
language and communication are as relevant as they were from the creation of humanity. The only variation is that it is a technology-driven in these modern times as we rely on various means to relay our messages and that capacity to transmit our feelings through various means and in this through symbols, emoticons and emoji. (Kazmai et al., 2019) which has diversified the ways we write and communicate through pictorial cue that substitute words and applies a more effective form of communication, thereby creating a different global language (Evans, 2014). Supporting this assertion, (Alshenqeeeti. 2019) pointed out that there is the universal application of emojis that opens up the reality that it may be on its way to a global language that will add to intercultural message transparency.

The popularity of emojis as a universal language has been extended since it was created in Japan in the 1990s and has been adopted into the Oxford English dictionary lexicons and was crowed as the top trending word by The Global Language monitor because its global acceptance and cultural acceptance by diverse groups. (Bennet, 2014). This situation is a pointer that semiotics through emoji has gained worldwide recognition as it has gone into the English lexicon to be recognised as a word that could be applied in communication and writing. This advanced with the advent of computers which can manipulate images and transmit them as messages as quickly as possible, without this possibility, there would not have progressed in the transmission of images across cultural boundaries this simplified semiotics and idealised it to a central concept that has been deployed by humanity and images were integrated into human communication and global world of images and pictures. According to Codognet (2002), This makes concrete the well-known dream of Leibniz of a universal language that would be both a lingua characteristica allowing the "perfect" description of knowledge by exhibiting the "real characters" of concepts and things... and making it possible for the mechanisation of reasoning (P.1)

This suggests that the computer has advanced the application and concreteness the application of emojis and other diacritic marks that have been slow in taking a prominent position in communication and editing. This impact of the computer has made semiotic communication popular around the world that has threatened to dissolve the mainstream culture of dialogue and writing. It attacked cultural domination and hegemony and developed its independent discourse pattern in the communication network. In consonance with this position, Xie (2019) pointed out that Emoticons are one of the essential means for contemporary youth to communicate and disseminate information in mass media. In recent years, the new network emoticons have swept through various chatting apps and platforms such as Wechat, Microblog, Line, Facebook, Twitter, and Instagram. The discourse that integrates visual symbols and linguistic symbols make our communication smoother and more vivid and help us avoid the embarrassment and misunderstanding that may occur in communication. (p.1)

This harps on the understanding that semiotic is a universal language through deployment in the social media have provided much freedom to the internet generation that has used that independence to push the global communication and acceptance of semiotics. It has changed the current landscape of conventional writing and communication to logographic and ideographic, typically designed to inform the recipient of the encoders intention or emotional situation (Joel.2018; Flavelle.2017) thereby making easily comprehensible from one language to another and from one culture to another as the convention does not regulate them.

\section{MATERIALS AND METHOD}

\section{Procedure}

This study was conducted through a survey of digital natives in Nigeria aged 15-20 years, with an estimated population of $82,000,000$. The sample size was $(n=267)$ in 2020 based on the calculation of the online sample size, 95\% confidence and 6\% intervals. Two hundred and sixty-seven responses were collected from public and private universities in Nigeria, i.e. 100 per cent. The rationale for the use of this sampling method is that the researcher believes that the sample chosen is the representation of the undergraduate population from the different departments of these universities for different coverage. Through a purposeful and snowball approach, respondents were contacted via email and WhatsApp to click a link to the online quasi-structured questionnaire via Google Form, WhatsApp and Facebook. Findings 
were analyzed using Nvivo and Excel software. The processes of this research involved four main steps: (i) documentation of subjects, (ii) strategy and development of the research instruments, (iii) carrying out of research, and (iv) data gathering and evaluation.

\section{The First Step Is to Know the Precise Information Needed to Carry Out the Research}

The second step was the design and development of the research instrument. Before the process, the researcher gathered information about emoticons, semiotics, emojis, smileys and other symbols that were crucial to the construction of the mechanisms. The third step has been

Conduct the survey, which was conducted within four weeks, involving a total of $(n=267)$

Graduate students from Nigerian universities. The population was chosen specifically for the survey because it represented the digital natives who are the recurrent users of the new media. The goal was to examine respondents' perceptions of semiotics in the context of formal communication.

Part of the research was to provide technical assistance during the appraisal. The concluding step was to analyze the data gathered. The researcher gathered the survey results of ten research questions made up of five research questions for question one and also five research questions for question two for gathering data from $(n=267)$ students using one set of online questionnaire. The purpose of using online questionnaire was due to the nature of the research, which was based on the new media and also for the ease of reach.

\section{Results}

Analysis of the survey is phased in line with the two research questions (RQ1 \& RQ2) of the study. RQ1 seeks to assess the extent of semiotic lettering intrusion into the formal patterns of communication and writing. $92.9 \%$, i.e. 248 respondents affirm understanding symbolic written communication like emoticons, emoji and memes. This survey tallies with the frequency of use of semiotics which peaks at $97.4 \%$ representing 260 respondents. On the possibility of semiotics in communication with persons with a different language, $69.3 \%$ affirm, $5.6 \%$ agree, while $24.7 \%$ are uncertain. The result is almost similar to the use of semiotics to converse with non-English speaking persons. The response curve takes a twist on the question of whether semiotics should be blended into formal or written communication; $25.1 \%$ agree, $46.1 \%$ disagree and 28.8 are indifferent.

TABLE 1

\section{COUNT OF RESPONSE ON ALL QUESTIONS IN RQ1}

\begin{tabular}{|l|l|l|l|l|l|l|}
\hline RESEARCH QUESTION ONE & \multicolumn{2}{|l|}{ AGREE } & \multicolumn{2}{|l|}{ DISAGREE } & \multicolumn{2}{|l|}{ INDIFFERENT } \\
\hline $\begin{array}{l}\text { 1. Do you understand symbolic writing like } \\
\text { emoticons, emojis, and memes? }\end{array}$ & 248 & $93 \%$ & 2 & $0.7 \%$ & 18 & $6.7 \%$ \\
\hline $\begin{array}{l}\text { 2. Do you sometimes communicate using } \\
\text { symbols like emoticons, emojis, and memes? }\end{array}$ & 260 & $97.4 \%$ & 0 & 0 & 7 & $2.6 \%$ \\
\hline $\begin{array}{l}\text { 3. Could symbols like emoticons, emojis, } \\
\text { and memes be used to communicate with } \\
\text { someone who does not understand your } \\
\text { language? }\end{array}$ & 185 & $69.3 \%$ & 15 & $5.6 \%$ & 66 & $24.7 \%$ \\
\hline $\begin{array}{l}\text { 4. Could they be used to communicate with } \\
\text { someone who does not understand the } \\
\text { English Language? }\end{array}$ & 182 & $68.2 \%$ & 18 & $6.7 \%$ & 67 & $25.1 \%$ \\
\hline $\begin{array}{l}\text { 5. Do you think they should be blended into } \\
\text { regular and formal written communication } \\
\text { pattern? }\end{array}$ & 67 & $25.1 \%$ & 123 & $46.1 \%$ & 77 & $28.8 \%$ \\
\hline
\end{tabular}

Source: Field Survey, 2020 


\section{FIGURE 3 \\ SUMMARY OF RQ1 - ASSESSING DIGITAL NATIVES USE OF SEMIOTICS IN FORMAL COMMUNICATION}

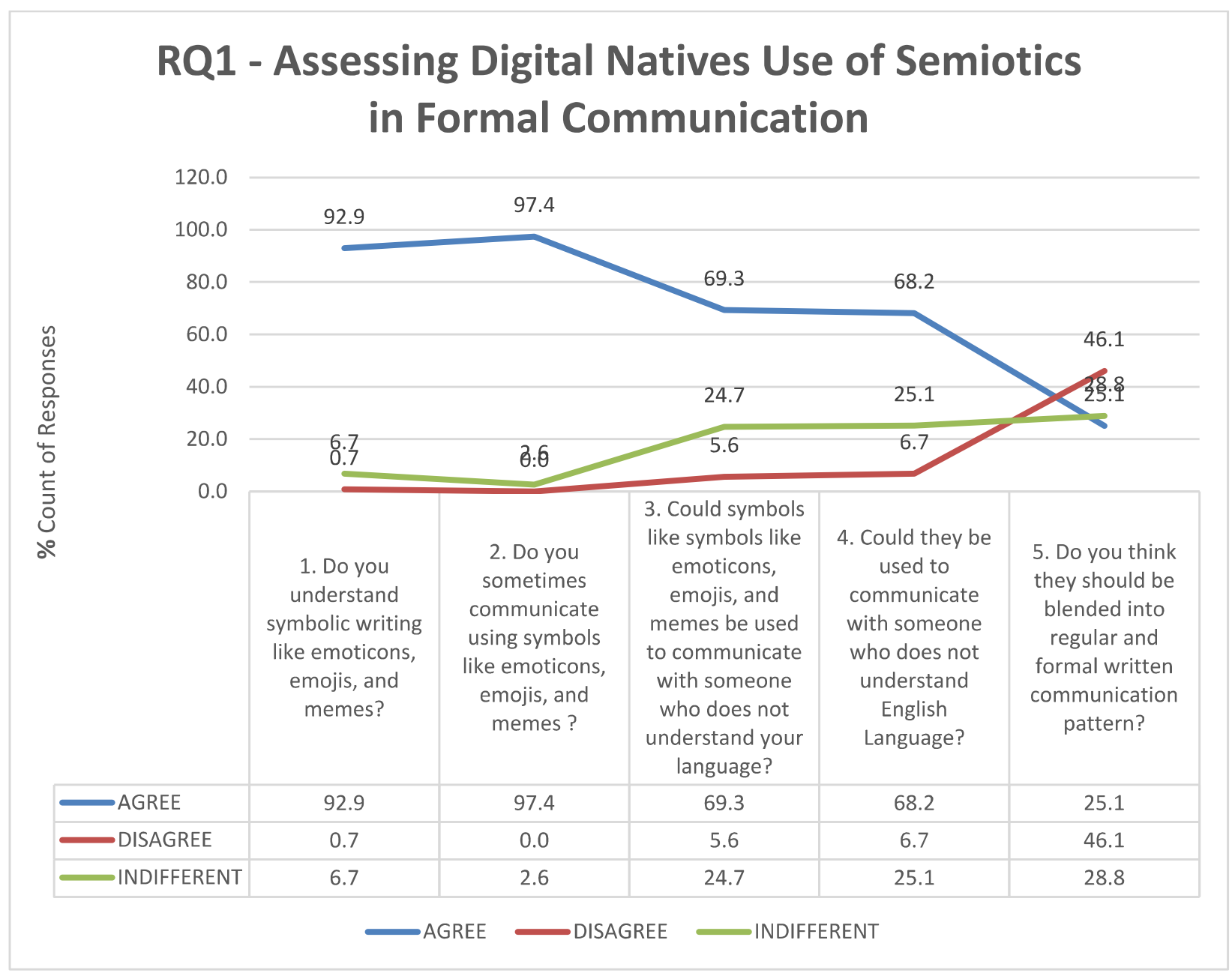

The chart above shows the trend in the use of frequency semiotics and the differences in perception of use with non-English persons or persons of different language. The funnel is even more apparent on the idea of blending semiotics with written \& formal communication. See figure 4 for the funnel trend. 


\section{FIGURE 4 \\ SUMMARY OF RQ1 SHOWING FUNNEL TREND IN PERCEPTION TO USE ON SEMIOTIC IN FORMAL COMMUNICATION AND WRITING}

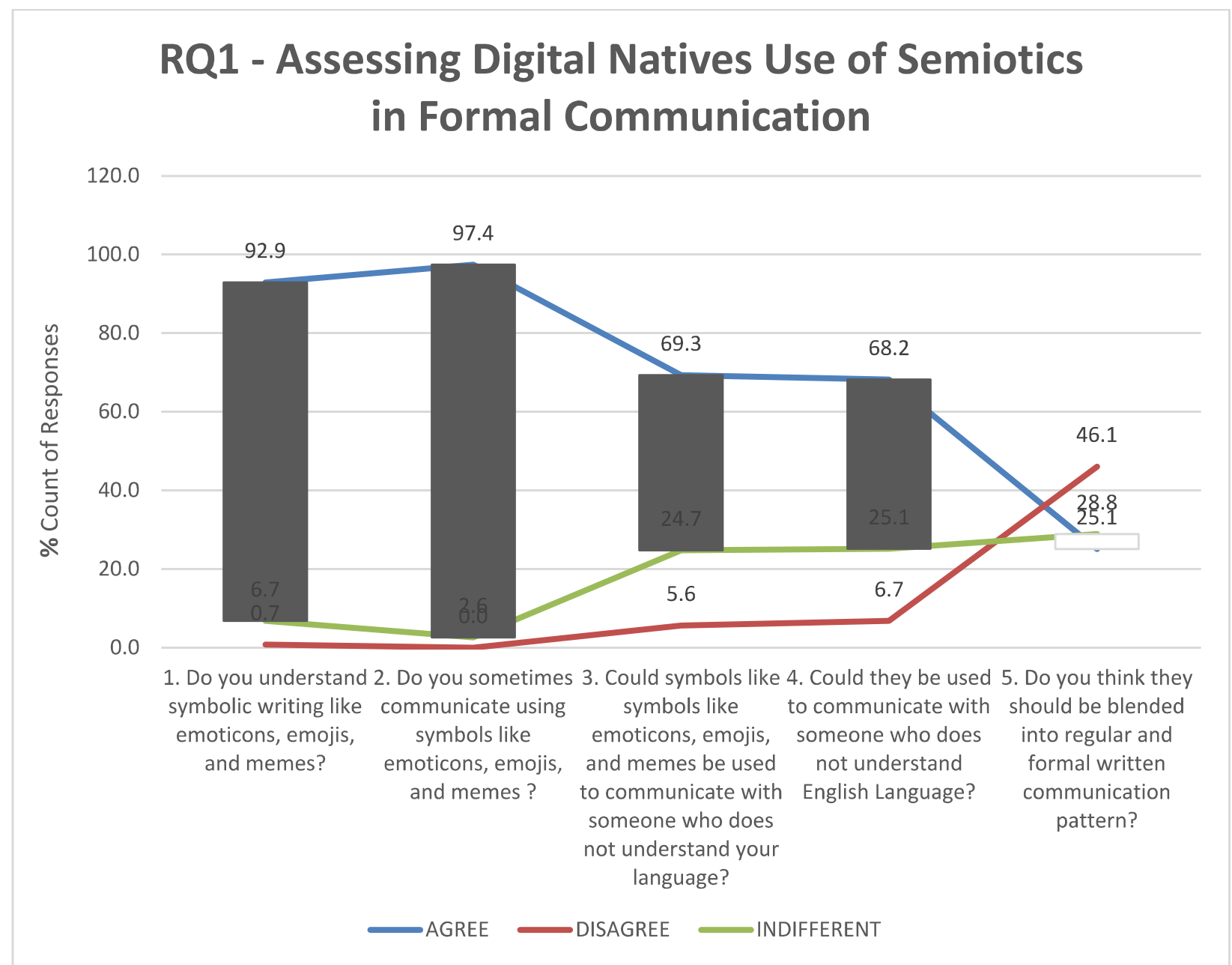

Source: Field Survey, 2020

Research Question 2 (RQ2), consists of five closed and two open-ended questions. It attempts to assess the impact and future of semiotics in written communication among the digital natives in Nigeria. See the summary of findings of the closed questions.

TABLE 2

COUNT OF RESPONSE ON ALL QUESTIONS IN RQ2

\begin{tabular}{|l|c|c|c|c|c|c|}
\hline RESEARCH QUESTION TWO & \multicolumn{2}{|l|}{ AGREE } & \multicolumn{2}{|l|}{ DISAGREE } & \multicolumn{2}{|l|}{ INDIFFERENT } \\
\hline $\begin{array}{l}\text { Do you consider the use of symbols, like } \\
\text { emoticons, emojis, and memes, a convenient } \\
\text { alternative means of writing? }\end{array}$ & 132 & $49.4 \%$ & 82 & $30.7 \%$ & 53 & $19.9 \%$ \\
\hline $\begin{array}{l}\text { Does it make communication more expressive } \\
\text { and free? }\end{array}$ & 219 & $82.0 \%$ & 18 & $6.7 \%$ & 30 & $11.2 \%$ \\
\hline
\end{tabular}




\begin{tabular}{|l|l|l|l|l|l|l|}
\hline $\begin{array}{l}\text { Do you consider semiotics (emoticons, emojis, } \\
\text { and memes) a threat to the standard or formal } \\
\text { ways of writing? }\end{array}$ & 73 & $27.3 \%$ & 150 & $56.2 \%$ & 44 & $16.5 \%$ \\
\hline $\begin{array}{l}\text { Does the use of symbols like emoticons, emojis, } \\
\text { and memes represent your social-cultural context } \\
\text { fully (i.e. Social habits, environment, patterns, } \\
\text { beliefs, etc.)? }\end{array}$ & 78 & $29.2 \%$ & 123 & $46.1 \%$ & 66 & $24.7 \%$ \\
\hline $\begin{array}{l}\text { Do you think that it may become a global means } \\
\text { of communicating in writing? }\end{array}$ & 104 & $39.0 \%$ & 87 & $32.6 \%$ & 76 & $28.5 \%$ \\
\hline
\end{tabular}

Source: Field Survey, 2020

See figure 3 indicating that $49.4 \%$ of the respondents consider the semiotics as an alternative means of writing. $82 \%$ opined it makes communication more expressive. $56.2 \%$ of the digital natives also indicated that semiotics is not a threat to formal writing; $27.3 \%$ consider it a threat, while $16.5 \%$ are indifferent. Furthermore, $46.1 \%$ feel that the use of semiotics does not necessarily represent their socio-cultural context fully. $29.2 \%$ say it serves them. However, 24.7 are indifferent. On the question of emoticons, emojis and memes becoming a global means of written communication, $39 \%$ agree to its potential, $32.6 \%$ disagree, while $28.5 \%$ were indifferent.

FIGURE 5

\section{SUMMARY OF RQ2 - IMPACT ASSESSMENT AND FUTURE OF SEMIOTICS IN WRITTEN} COMMUNICATION AMONG THE DIGITAL NATIVES IN NIGERIA

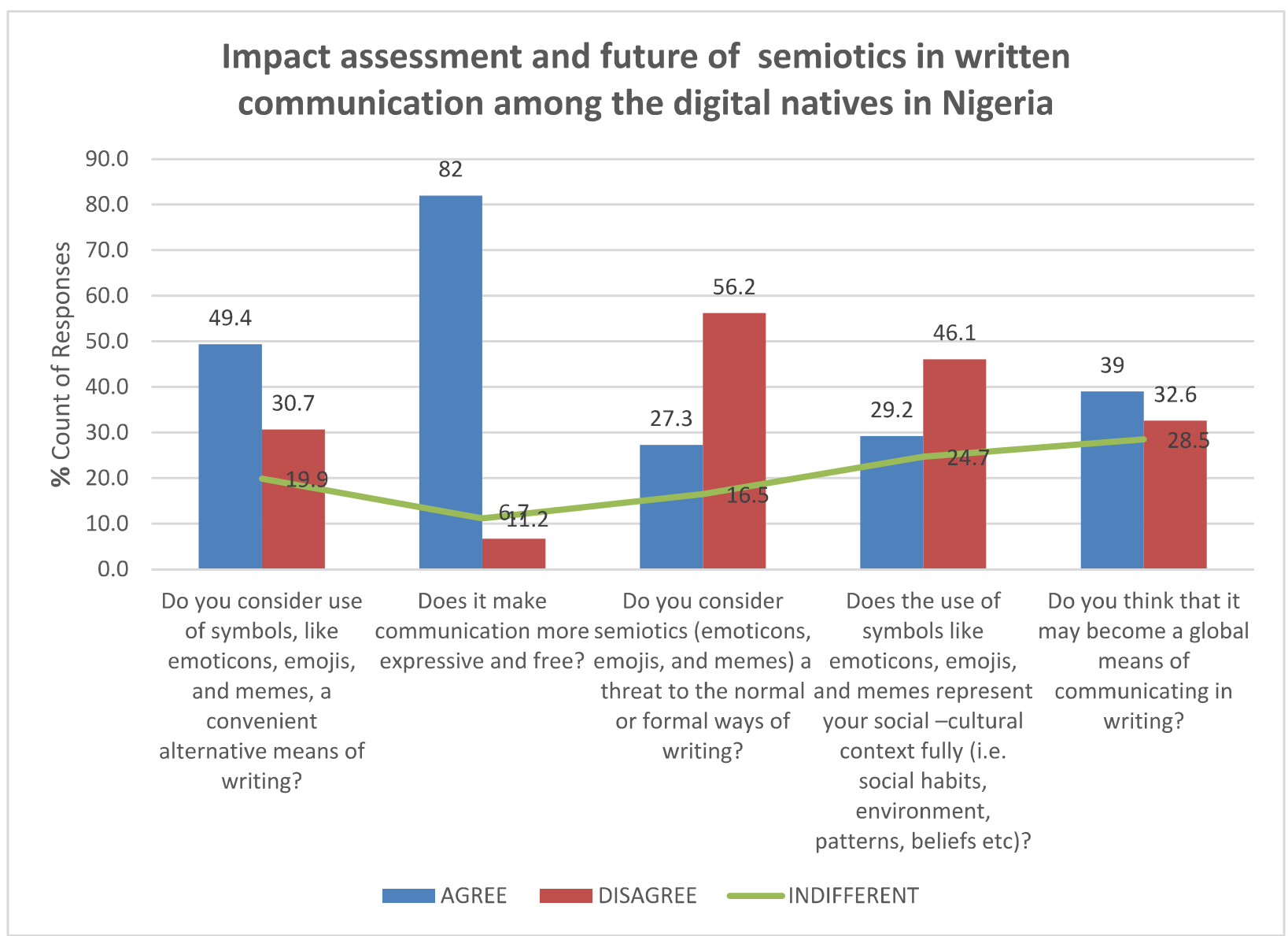

Source: Field Survey, 2020. 
The respondents went further to explain why semiotics is a threat or not. They also gave short texts on why semiotics may or may become part of written communication. This qualitative data was coded using Nvivo software.

FIGURE 6

RQ 2- CODING/THEMES OF QUALITATIVE RESPONSES ON SEMIOTICS AS NOT A THREAT TO FORMAL WRITING

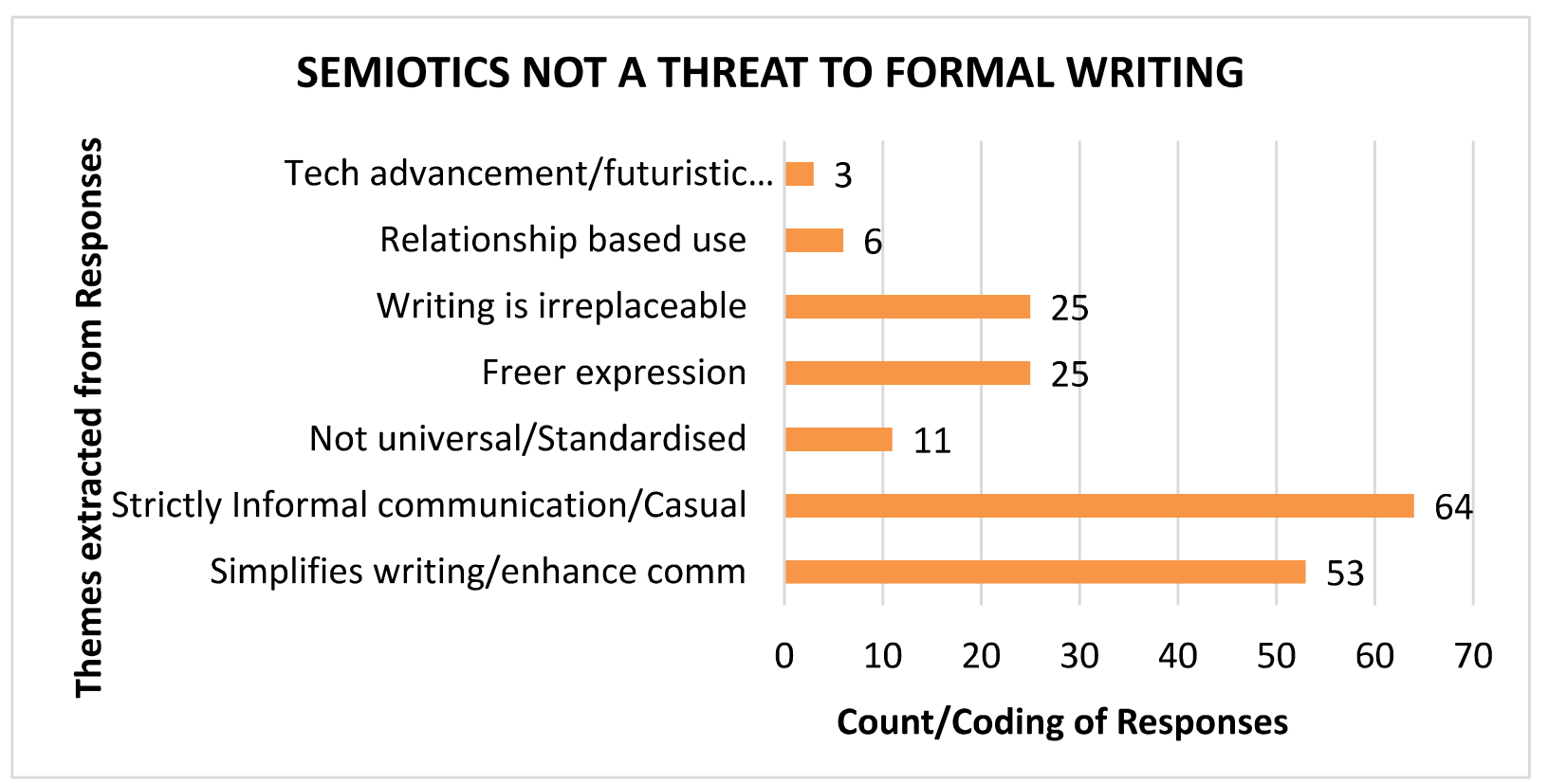

Source: Field Survey, 2020 
FIGURE 7

RQ 2- CODING/THEMES OF QUALITATIVE RESPONSES ON SEMIOTICS AS NOT A THREAT TO FORMAL WRITING

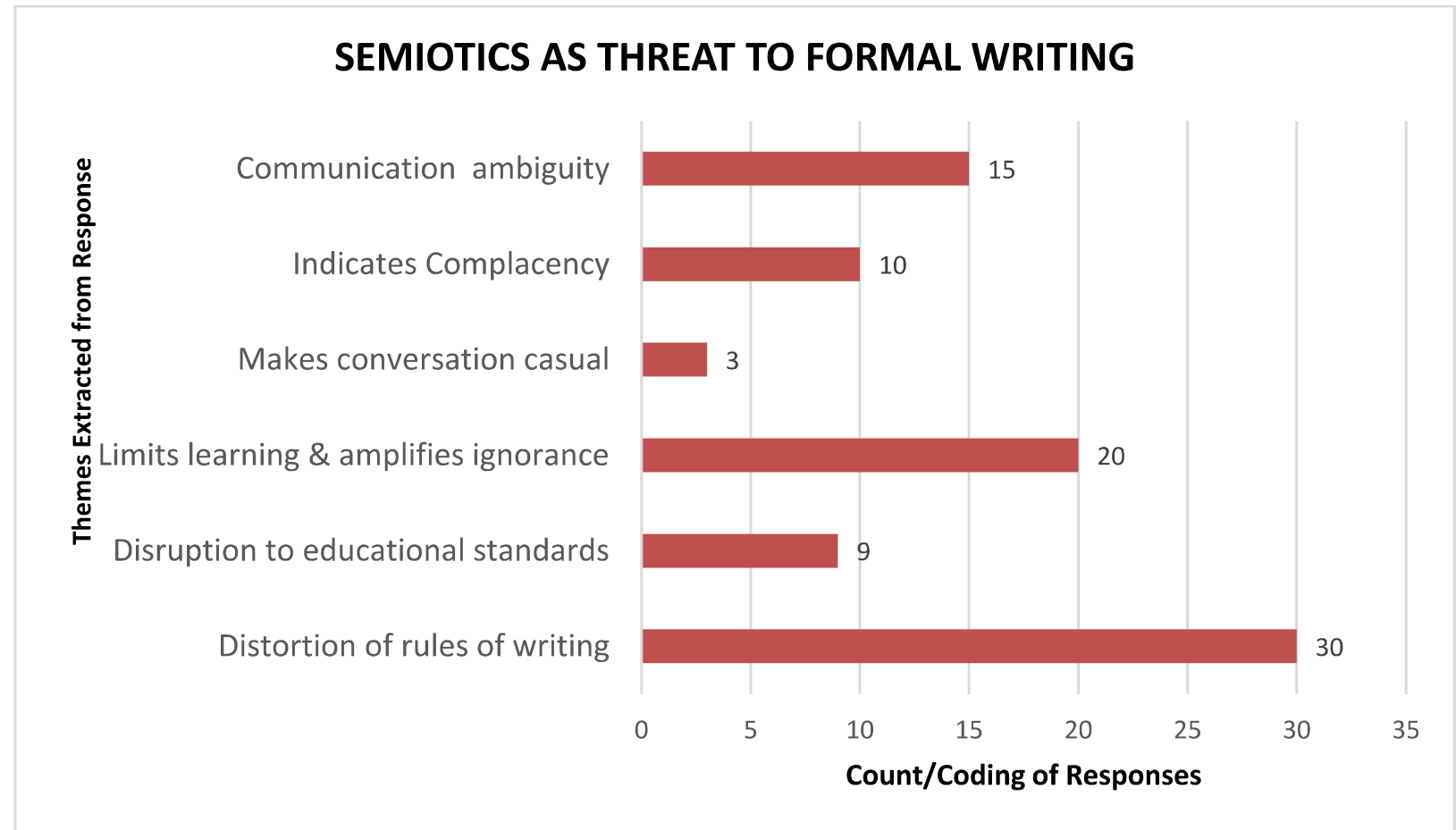

Source: Field Survey, 2020

FIGURE 8

RQ 2- CODING/THEMES OF QUALITATIVE RESPONSES ON SEMIOTICS BECOMING A GLOBAL MEANS OF COMMUNICATING IN WRITING

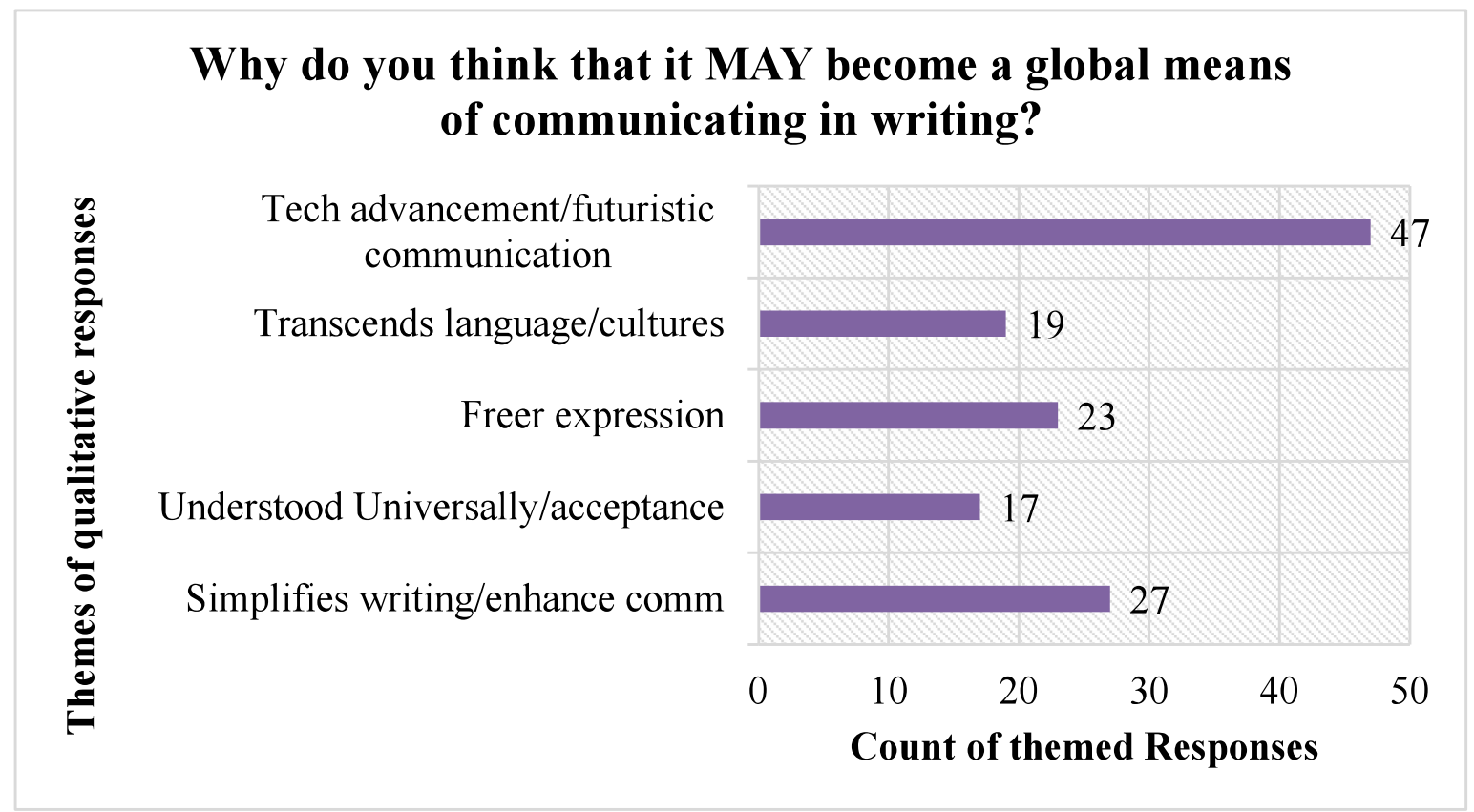

Source: Field Survey, 2020 


\section{FIGURE 9 \\ RQ 2- CODING/THEMES OF QUALITATIVE RESPONSES ON SEMIOTICS NOT \\ BECOMING A GLOBAL MEANS OF COMMUNICATING IN WRITING}

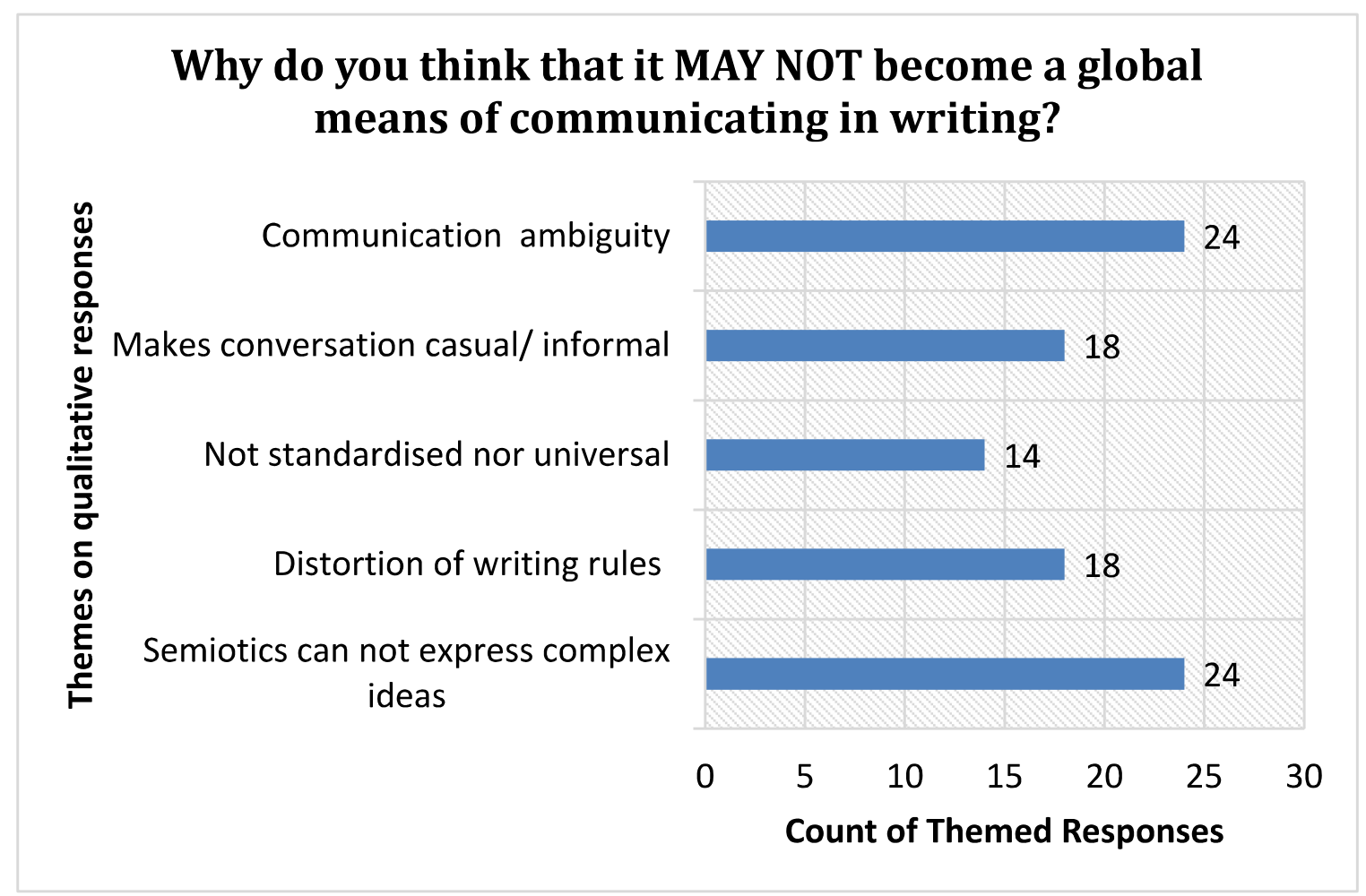

Source: Field Survey, 2020

\section{DISCUSSION}

Findings on RQ1 consists of five questions an investigated extent of semiotic lettering intrusion in formal communication and writing by digital natives. Results indicated that majority, over $92 \%$ of the digital natives understand, use and communicate using memes, emoticons and emojis. Furthermore, over $68 \%$ agreed to the potential of using memes, emoticons and emojis for communicating with persons of other language or non-English speaking climes. Whereas $<6.7 \%$ disagree and $<25.1 \%$ are indifferent. The trend on RQ1 slopes drastically on the possibility or potential for a blend of memes, emoticons and emojis in formal written communication with $25.1 \%$ agreeing, 46.1 disagreeing and $28.8 \%$ being indifferent.

So while the digital natives understand and effectively use semiotics, they are inconclusive about it being integrated into formal written communication. RQ2 findings shed some light on the possible reasons for this. RQ2, consisting of five mixed questions, integrated quantitative and qualitative sections and drills deeper to investigate how semiotics makes communication convenient among the digital generation and the potential of it being universal.

While as high as $82 \%$ consider the use of memes, emoticons and emojis as more expressive in communication, $<50 \%$ agreed that it is a convenient alternative means of writing, $30.7 \%$ disagreed to its convenience for writing, and $11.2 \%$ were indifferent.

On the question of memes, emoticons and emojis being a threat or not to formal written communication, opinions were bifurcated and analysed for themes. In the first part, i.e. semiotics NOT being a threat to formal writing, it was opined that semiotics simply enhances communication, allows freer expressions, simplifies writing, is primarily casual and cannot replace formal written communication patterns. It is also 
not a threat because it is neither standardised yet nor universal. Finally, it is an indication of language evolution, is amplified by technological advancement and is futuristic in scope.

In response to why semiotics is potentially a threat to written communication, the themes drawn from respondents discourse were that the use of memes, emojis and emoticons could lead to a distortion of writing rules, disruption in education standards and trivialise formal discussion. Furthermore, there is the potential for frequency in use to limit learning capacity of students, fuel complacency, amplify ignorance of the concepts (complex or not) and finally lead to miscommunication due to ambiguity of symbolisation.

A test of a standard deviation on the themes on both parts of this question is as follows:

- Semiotics not a threat to written communication: $\mathrm{n}=187$, Mean $=26.71$, Standard Deviation $=23.54$

- Semiotics as a threat to written communication: $\mathrm{n}=87$, Mean $=14.5$, Standard Deviation $=$ 9.52

The above analysis indicates a conclusion of semiotics as not being a threat to formal written communication.

The penultimate question was whether the use of symbols like emoticons, emojis, and memes represents their social-cultural context fully (i.e. Social habits, environment, patterns, beliefs, among others). With this, $46.1 \%$ disagreed, $29.2 \%$ agreed, while $24.7 \%$ were indifferent. The researchers consider this outcome to be due to the ubiquity of the internet, social media, globalisation, ease of global connections and social influence across platforms despite social-cultural contexts

The final question under RQ2 tested the perception of the potential of semiotics being universal and a global means of communicating in writing. Again, responses were bifurcated, and themes drawn from respondents discourse are listed and analysed below. On whether semiotics (memes, emojis and emoticons) may become a global means of written communication, pro-themes include that semiotics transcends languages/cultures and has received large-scale acceptance. This acceptance was tied to the worldwide trend, technological advancements, the ubiquity of social media applications (typically embedding semiotics) and gives rise to the idea of language evolution and futuristic communication. Furthermore, semiotics enhance writing and simplifies communication.

The second part of the question considers that semiotics MAY NOT become a global means of written communication because it makes the discourse casual or informal and does not suffice in expressing complex ideas. Furthermore, semiotics is not necessarily standardised globally; they may lead to distortion of writing rules and ultimately miscommunication due to ambiguous and varied interpretations of symbolic writings or expressions.

The mean and standard deviation of both parts of the question are as follows:

- Semiotics may become a global means of writing: $\mathrm{n}=133$, Mean $=26.6$, Standard Deviation $=12.03$

- Semiotics may NOT become a global means of writing: $n=74$, Mean $=19.6$, Standard Deviation $=4.34$

The conclusion from the analysis above indicates that semiotics may become a global means of writing. This is instructive as mobile devices and other hardware gadgets leverage semiotics in presenting their applications, user guides, onboarding, feedback reviews etc, enhancing ease of use, communications and adaptability.

\section{RECOMMENDATION AND FUTURE STUDIES}

This study investigated the use and advancement of semiotics in the communication of college students in Nigeria. As a result of our findings, it would be inconclusive to have a definite opinion about the use of semiotics by the digital natives, thus the call for further studies.

Our findings indicate that the reception of semiotics is polysemic, the digital natives did not regard semiotics as a threat to formal writing, even though they are adept to the usage in communication, could this be due to hegemony, stereotypes or any other reason? 
Another area requiring further study is an understanding of why respondents consider semiotics as having potential for universality and a global means of communication which transcend immediate sociocultural interactions. Nevertheless, they were reluctant about its integration of into formal learning and writing scenarios. The future of research in this field could also concentrate on requirements and need for a universal language in contemplation of globalised learning. This research finds that the power of symbolic writing among digital natives is under-estimated. Their high frequency in the use of semiotics in communicativity and their tendency to advance with trends and push ideas via the internet portends a research gap. Furthermore, there is dissonance arising from their prior knowledge of the rules of writing taught in school and the preponderance of semiotics (calling for use) in current communication applications and platforms, including social media are now being integrated to formal learning.

\section{REFERENCES}

Alshenqeeti, H. (2016). Researchgate. Retrieved August 14, 2020, from www.researchgate.net.

Bennet, W.L. (2014). Changing society, changing media systems. The challenges of communication, theory and education. Seatle, Washington: University of Washington.

Codognet, P. (2002). Researchgate. Retrieved August 14, 2020, from www.researchgate.net.

Danesi, M. (2017). The rise of visual language in the age of the internet. London: Bloomsbury Publishers.

Danesi, M. (2017). The semiotics of Emoji: The rise of visual language in the age of the internet. London: Bloomsbury Publishers.

Eco, U. (1979). A theory of semiotics. In U. Eco, A theory of semiotics. Indiana: Indiana University Press. Evans, V. (2014). The language myth. Cambridge: Cambridge University Press.

Feldman, J. (2012). Symbolic representation of a probabilistic world. Cognition, 123(1), 61-83.

Kazmi, A., Rana, A., Anjum, U., \& Khan, M. (2019). A picture is worth a thousand words, and so is an emoji? Emojification of languages. A pragmatic analysis of Facebook discourse. Retrieved from https:doc.lib.purdue.edu/plcc/2019/3.

Nölle, J., Staib, M., Fusaroli, R., \& Tylén, K. (2018). The emergence of systematicity.How environment and communicative factors shape a novel communication. Cognition, 181, 93-104.

Paul, P. (2014). Researchgate. Retrieved August 14, 2020, from www.researchgate.net/publications.

Richard, N. (2020). Thoughtco.

Stanford Encyclopedia of philosophy. (2010). Peirce theory of sign. Retrieved August 14, 2020, from www.plato.Stanford.edu/entries/peice-semiotics.

Xie, C. (2019). Construction of emoticon discourse: Advances in social science education and humanities research. 2nd International Conference on Education, Economics and Social Science (ICEESS 2019) (pp. 153-156). Singapore: Atlantis Press.

Yakin, H.S.N., \& Totu, A. (2014). Semiotics perspectives of Peirce and Saussure: A brief comparative study. Prodesia, 155, 4-8. 


\section{APPENDICES}

\section{Tables}

- Table 2: Count of response on all questions in RQ1. Source: Field Survey, 2020

- Table 3: Count of response on all questions in RQ2. Source: Field Survey, 2020

\section{Figures}

- Figure 2: The relationship between the three theories. (this study's construct)

- Figure 3: Summary of RQ1 showing funnel trend in perception to use on semiotic in formal communication and writing.

- Figure 4: Summary of RQ2 - Impact assessment and future of semiotics in written communication among the digital natives in Nigeria

- Figure 5: Summary of RQ2 - Impact assessment and future of semiotics in written communication among the digital natives in Nigeria.

- Figure 6: RQ 2- Coding/Themes of Qualitative Responses on Semiotics as NOT a threat to formal writing.

- Figure 7: RQ 2- Coding/Themes of Qualitative Responses on Semiotics as a threat to formal writing.

- Figure 8: RQ 2- Coding/Themes of Qualitative Responses on Semiotics becoming a global means of communicating in writing.

- Figure 9: RQ 2- Coding/Themes of Qualitative Responses on Semiotics NOT becoming a global means of communicating in writing. 